\title{
Evolution of Acoustic Communication Between Two Cooperating Robots
}

\author{
Elio Tuci and Christos Ampatzis \\ CoDE-IRIDIA, Université Libre de Bruxelles - Bruxelles - Belgium \\ \{etuci, campatzi\}@ulb.ac.be
}

\section{The fitness function}

During evolution, each genotype is translated into a robot controller (i.e., modules $M_{C}$ and $M_{M}$ see [1]), and cloned in each agent. Then, the two robot group is evaluated two times in each environment type $E_{11}, E_{00}, E_{01}$, and $E_{10}$, for a total of eight trials. Note that the sequence order of the environment type experienced by the robots - randomly chosen at the beginning of each generation - has a bearing on the overall performance of the group since the robots' controllers are reset only at the beginning of the first trial. Each trial differs from the others in the initialisation of the random number generator, which influences the robots' starting position and orientation anytime the robots are positioned, and the noise added to motors and sensors. The robots are randomly placed in the arena at the beginning of the first trial and repositioned in subsequent trials following an unsuccessful one. Within a trial, the robots life-span is 90 simulated seconds (900 simulation cycles). A trial is terminated earlier in case a robot crashes with the arena walls, or if the group successfully accomplishes its task. For each trial $e \in[1,8]$, the group is rewarded by an evaluation function which seeks to assess the ability of the robots to open the revolving door located at the centre of the arena. This requires the robots to be able to determine the nature of the environment (i.e., $E_{11}, E_{00}, E_{01}$, or $E_{10}$ ) by using acoustic communication. The final fitness $F$ attributed to a group controlled by a specific genotype is the average group score over a set of eight trials, and it is computed as follows:

$$
\begin{aligned}
& F=\left(\frac{\sum_{e=1}^{8} F M_{e}}{8}\right)+F C ; \quad F M_{e}=\frac{\sum_{r=1}^{2}\left(\psi_{r}+f m_{r} \kappa_{r} \rho_{r}\right)}{2}, F M_{e} \in[0,2.4] ; \\
& F C= \begin{cases}0, & \text { if } \frac{\left(\sum_{e=1}^{8} \sum_{r=1}^{2} f c_{e r}\right)}{2} \leq 0 ;, F C \in[0,1.0] \\
\frac{\sum_{e=1}^{8} \sum_{r=1}^{2} f c_{e r}}{2}, & \text { otherwise }\end{cases}
\end{aligned}
$$

$\psi_{r}=0$ if robot $r$ didn't terminate the first phase of a trial, otherwise $\psi_{r}=1$. $\rho_{r}=\frac{1}{5}$ if robot $r$ collided with the arena walls, otherwise $\rho_{r}=1$. $f m_{r}=$ $1.0-\left(d_{r L_{i}}\right)$ with $d_{r L_{i}}$ corresponding to the normalised distance between the robot $r$ and the light $L_{i}$. During the first phase of a trial $i=1$ for the robot $r$ 
located in the lower side of the arena, and $i=2$ for the robot $r$ located in the upper side of the arena. During the second phase of a trial, $i=1$ for the robot $r$ located in the upper side of the arena, and $i=2$ for the robot $r$ located in the lower side of the arena. $\kappa_{r}=1$ if robot $r$ didn't terminate the first phase of a trial, or if, after having done so, it exerts pushing forces in the rotational direction of the revolving door. $\kappa_{r}=0.5$ if robot $r$, after having terminated the first phase of a trial, it exerts pushing forces in the anti-rotational direction of the revolving door. $f c_{e r}=\frac{\sum_{s=\left(t_{c}\right)}^{T}\left(P_{s-t c}\right)}{T-t_{c}}$, where $t_{c}$ corresponds to the simulation cycles at 10 seconds after the end of the first part of the task, $T$ corresponds to the simulations cycles at the end of the trial $e$ and

$$
P_{s-t c}=\left\{\begin{array}{l}
+1 \text { if }\left(\left(E_{11} \vee E_{00}\right) \wedge\left(S_{C}=0\right)\right) \vee\left(\left(E_{10} \vee E_{01}\right) \wedge\left(S_{C}=1\right)\right) \\
-1 \text { if }\left(\left(E_{11} \vee E_{00}\right) \wedge\left(S_{C}=1\right)\right) \vee\left(\left(E_{10} \vee E_{01}\right) \wedge\left(S_{C}=0\right)\right)
\end{array}\right.
$$

In other words, during the first phase of a trial, $F M_{e}$ rewards the robots for approaching the light at the corresponding side of the arena (i.e., $L_{1}$ for robot in the lower side; $L_{2}$ for robot in the upper side). During the second phase of a trial, $F M_{e}$ rewards the robots for approaching the opposite side of the arena. $F C$ rewards the robots for setting the state of the fifth neurons (i.e., $\left.y_{5}\right)$ of $M_{C}$ so that (a) $S_{C}$ results equal to 0 during the second phase of trials in $E_{00}$ and $E_{11}$; (b) $S_{C}$ results equal to 1 during the second phase of trials in $E_{10}$ and $E_{01}$.

Note that $F$ doesn't refer anyhow to signalling behaviour. $F$ rewards the robots for accomplishing the task. However, due to the nature of the task, the robots can be successful only if they coordinate their actions using the sound signalling system. By leaving signalling behaviour out of the fitness function, we clean our model from preconceptions concerning what (i.e., semantics) and how (i.e., syntax) successful group communicates, and we let evolution determine the characteristics of the communication protocol.

\section{References}

1. E. Tuci and C. Ampatzis. Evolution of acoustic communication between two cooperating robots. Technical Report TR/IRIDIA/2007-013, CoDE-IRIDIA, Univeristé Libre de Bruxelles, June 2007. The technical report can be found at http://iridia.ulb.ac.be/ etuci/publications.html. 